\title{
Functional equations and inequalities in paranormed spaces
}

\author{
Choonkil Park ${ }^{1}$ and Jung Rye Lee $2^{2^{*}}$
}

"Correspondence: jrlee@daejin.ac.kr ${ }^{2}$ Department of Mathematics, Daejin University, Kyeonggi, 487-711, Korea

Full list of author information is available at the end of the article

\begin{abstract}
Using the fixed point method and the direct method, we prove the Hyers-Ulam stability of an additive functional equation, a quadratic functional equation, a cubic functional equation and a quartic functional equation in paranormed spaces. Furthermore, we prove the Hyers-Ulam stability of functional inequalities in paranormed spaces by using the fixed point method and the direct method.
\end{abstract} MSC: Primary 35A17; 47H10; 39B52; 39B72

Keywords: Jordan-von Neumann functional equation; Hyers-Ulam stability; paranormed space; fixed point; functional equation; functional inequality

\section{Introduction and preliminaries}

The concept of statistical convergence for sequences of real numbers was introduced by Fast [1] and Steinhaus [2] independently, and since then several generalizations and applications of this notion have been investigated by various authors (see [3-7]). This notion was defined in normed spaces by Kolk [8].

We recall some basic facts concerning Fréchet spaces.

Definition 1.1 [9] Let $X$ be a vector space. A paranorm $P: X \rightarrow[0, \infty)$ is a function on $X$ such that

(1) $P(0)=0$;

(2) $P(-x)=P(x)$;

(3) $P(x+y) \leq P(x)+P(y)$ (triangle inequality)

(4) If $\left\{t_{n}\right\}$ is a sequence of scalars with $t_{n} \rightarrow t$ and $\left\{x_{n}\right\} \subset X$ with $P\left(x_{n}-x\right) \rightarrow 0$, then $P\left(t_{n} x_{n}-t x\right) \rightarrow 0$ (continuity of multiplication).

The pair $(X, P)$ is called a paranormed space if $P$ is a paranorm on $X$.

The paranorm is called total if, in addition, we have

(5) $P(x)=0$ implies $x=0$.

A Fréchet space is a total and complete paranormed space.

The stability problem of functional equations originated from a question of Ulam [10] concerning the stability of group homomorphisms. Hyers [11] gave the first affirmative partial answer to the question of Ulam for Banach spaces. Hyers' theorem was generalized by Aoki [12] for additive mappings and by Rassias [13] for linear mappings by considering an unbounded Cauchy difference. A generalization of the Rassias theorem was obtained by 
Găvruta [14] by replacing the unbounded Cauchy difference by a general control function in the spirit of Rassias' approach.

In 1990, Rassias [15] during the 27th International Symposium on Functional Equations asked the question whether such a theorem can also be proved for $p \geq 1$. In 1991, Gajda [16], following the same approach as in Rassias [13], gave an affirmative solution to this question for $p>1$. It was shown by Gajda [16], as well as by Rassias and Šemrl [17], that one cannot prove a Rassias-type theorem when $p=1$ ( $c f$. the books of Czerwik [18], Hyers, Isac and Rassias [19]).

The functional equation

$$
f(x+y)+f(x-y)=2 f(x)+2 f(y)
$$

is called a quadratic functional equation. In particular, every solution of the quadratic functional equation is said to be a quadratic mapping. A Hyers-Ulam stability problem for the quadratic functional equation was proved by Skof [20] for mappings $f: X \rightarrow Y$, where $X$ is a normed space and $Y$ is a Banach space. Cholewa [21] noticed that the theorem of Skof is still true if the relevant domain $X$ is replaced by an Abelian group. Czerwik [22] proved the Hyers-Ulam stability of the quadratic functional equation. The stability problems of several functional equations have been extensively investigated by a number of authors, and there are many interesting results concerning this problem (see [23-29]).

In [30], Jun and Kim considered the following cubic functional equation:

$$
\frac{1}{2} f(2 x+y)+\frac{1}{2} f(2 x-y)=f(x+y)+f(x-y)+6 f(x) .
$$

It is easy to show that the function $f(x)=x^{3}$ satisfies the functional equation (1.2), which is called a cubic functional equation, and every solution of the cubic functional equation is said to be a cubic mapping.

In [31], Lee et al. considered the following quartic functional equation:

$$
\frac{1}{2} f(2 x+y)+\frac{1}{2} f(2 x-y)=2 f(x+y)+2 f(x-y)+12 f(x)-3 f(y) .
$$

It is easy to show that the function $f(x)=x^{4}$ satisfies the functional equation (1.3), which is called a quartic functional equation, and every solution of the quartic functional equation is said to be a quartic mapping.

In [32], Gilányi showed that if $f$ satisfies the functional inequality

$$
\left\|2 f(x)+2 f(y)-f\left(x y^{-1}\right)\right\| \leq\|f(x y)\|,
$$

then $f$ satisfies the Jordan-von Neumann functional equation

$$
2 f(x)+2 f(y)=f(x y)+f\left(x y^{-1}\right) .
$$

See also [33]. Fechner [34] and Gilányi [35] proved the Hyers-Ulam stability of the functional inequality (1.4). 
Park, Cho and Han [36] proved the Hyers-Ulam stability of the following functional inequalities:

$$
\begin{aligned}
& \|f(x)+f(y)+f(z)\| \leq\left\|2 f\left(\frac{x+y+z}{2}\right)\right\|, \\
& \|f(x)+f(y)+f(z)\| \leq\|f(x+y+z)\|, \\
& \|f(x)+f(y)+2 f(z)\| \leq\left\|2 f\left(\frac{x+y}{2}+z\right)\right\| .
\end{aligned}
$$

Throughout this paper, assume that $(X, P)$ is a Fréchet space and that $(Y,\|\cdot\|)$ is a Banach space.

In this paper, we prove the Hyers-Ulam stability of the Cauchy additive functional equation, the quadratic functional equation (1.2), the cubic functional equation (1.2) and the quartic functional equation (1.3) in paranormed spaces by using the fixed point method and the direct method.

Furthermore, we prove the Hyers-Ulam stability of the functional inequalities (1.5), (1.6) and (1.7) in paranormed spaces by using the fixed point method and the direct method.

\section{Hyers-Ulam stability of the Cauchy additive functional equation}

Using the fixed point method and the direct method, we prove the Hyers-Ulam stability of the Cauchy additive functional equation in paranormed spaces.

Let $S$ be a set. A function $m: S \times S \rightarrow[0, \infty]$ is called a generalized metric on $S$ if $m$ satisfies

(1) $m(x, y)=0$ if and only if $x=y$;

(2) $m(x, y)=m(y, x)$ for all $x, y \in S$;

(3) $m(x, z) \leq m(x, y)+m(y, z)$ for all $x, y, z \in S$.

We recall a fundamental result in fixed point theory.

Theorem 2.1 [37, 38] Let $(S, m)$ be a complete generalized metric space, and let $J: S \rightarrow S$ be a strictly contractive mapping with a Lipschitz constant $\alpha<1$. Then, for each given element $x \in S$, either

$$
m\left(J^{n} x, J^{n+1} x\right)=\infty
$$

for all nonnegative integers $n$ or there exists a positive integer $n_{0}$ such that

(1) $m\left(J^{n} x, J^{n+1} x\right)<\infty, \forall n \geq n_{0}$;

(2) the sequence $\left\{J^{n} x\right\}$ converges to a fixed point $y^{*}$ of $J$;

(3) $y^{*}$ is the unique fixed point of $J$ in the set $W=\left\{y \in S \mid m\left(J^{n_{0}} x, y\right)<\infty\right\}$;

(4) $m\left(y, y^{*}\right) \leq \frac{1}{1-\alpha} m(y, J y)$ for all $y \in W$.

In 1996, Isac and Rassias [39] were the first to provide applications of stability theory of functional equations for the proof of new fixed point theorems with applications. By using fixed point methods, the stability problems of several functional equations have been extensively investigated by a number of authors (see [40-44]).

Note that $P(2 x) \leq 2 P(x)$ for all $x \in Y$. 
Theorem 2.2 Let $\varphi: X^{2} \rightarrow[0, \infty)$ be a function such that there exists an $\alpha<1$ with

$$
\varphi(x, y) \leq 2 \alpha \varphi\left(\frac{x}{2}, \frac{y}{2}\right)
$$

for all $x, y \in X$. Let $f: X \rightarrow Y$ be a mapping such that

$$
\|f(x+y)-f(x)-f(y)\| \leq \varphi(x, y)
$$

for all $x, y \in X$. Then there exists a unique Cauchy additive mapping $A: X \rightarrow Y$ such that

$$
\|f(x)-A(x)\| \leq \frac{1}{2-2 \alpha} \varphi(x, x)
$$

for all $x \in X$.

Proof Letting $y=x$ in (2.2), we get

$$
\|f(2 x)-2 f(x)\| \leq \varphi(x, x)
$$

and so

$$
\left\|f(x)-\frac{1}{2} f(2 x)\right\| \leq \frac{1}{2} \varphi(x, x)
$$

for all $x \in X$.

Consider the set

$$
S:=\{h: X \rightarrow Y\}
$$

and introduce the generalized metric on $S$ :

$$
m(g, h)=\inf \left\{\mu \in \mathbb{R}_{+}:\|g(x)-h(x)\| \leq \mu \varphi(x, x), \forall x \in X\right\},
$$

where, as usual, $\inf \phi=+\infty$. It is easy to show that $(S, m)$ is complete (see [45, Lemma 2.1]). Now we consider the linear mapping $J: S \rightarrow S$ such that

$$
J h(x):=\frac{1}{2} h(2 x)
$$

for all $x \in X$.

Let $g, h \in S$ be given such that $m(g, h)=\varepsilon$. Since

$$
\|J g(x)-J h(x)\|=\left\|\frac{1}{2} g(2 x)-\frac{1}{2} h(2 x)\right\| \leq \alpha \varphi(x, x)
$$

for all $x \in X, m(g, h)=\varepsilon$ implies that $m(J g, J h) \leq \alpha \varepsilon$. This means that

$$
m(J g, J h) \leq \alpha m(g, h)
$$

for all $g, h \in S$. 
It follows from (2.4) that $m(f, J f) \leq \frac{1}{2}$.

By Theorem 2.1, there exists a mapping $A: X \rightarrow Y$ satisfying the following:

(1) $A$ is a fixed point of $J$, i.e.,

$$
A(2 x)=2 A(x)
$$

for all $x \in X$. The mapping $A$ is a unique fixed point of $J$ in the set

$$
M=\{g \in S: m(f, g)<\infty\} .
$$

This implies that $A$ is a unique mapping satisfying (2.5) such that there exists a $\mu \in(0, \infty)$ satisfying

$$
\|f(x)-A(x)\| \leq \mu \varphi(x, x)
$$

for all $x \in X$;

(2) $m\left(J^{n} f, A\right) \rightarrow 0$ as $n \rightarrow \infty$. This implies the equality

$$
\lim _{n \rightarrow \infty} \frac{1}{2^{n}} f\left(2^{n} x\right)=A(x)
$$

for all $x \in X$;

(3) $m(f, A) \leq \frac{1}{1-\alpha} m(f, f f)$, which implies the inequality

$$
m(f, A) \leq \frac{1}{2-2 \alpha}
$$

This implies that the inequality (2.3) holds true.

It follows from (2.1) and (2.2) that

$$
\begin{aligned}
\|A(x+y)-A(x)-A(y)\| & =\lim _{n \rightarrow \infty} \frac{1}{2^{n}}\left\|f\left(2^{n}(x+y)\right)-f\left(2^{n} x\right)-f\left(2^{n} y\right)\right\| \\
& \leq \lim _{n \rightarrow \infty} \frac{2^{n} \alpha^{n}}{2^{n}} \varphi(x, y)=0
\end{aligned}
$$

for all $x, y \in X$. So, $A(x+y)-A(x)-A(y)=0$ for all $x, y \in X$. Thus $A: X \rightarrow Y$ is an additive mapping, as desired.

Corollary 2.3 Let $r$ be a positive real number with $r<1$, and let $f: X \rightarrow Y$ be a mapping such that

$$
\|f(x+y)-f(x)-f(y)\| \leq P(x)^{r}+P(y)^{r}
$$

for all $x, y \in X$. Then there exists a unique Cauchy additive mapping $A: X \rightarrow Y$ such that

$$
\|f(x)-A(x)\| \leq \frac{2}{2-2^{r}} P(x)^{r}
$$

for all $x \in X$. 
Proof Taking $\varphi(x, y)=P(x)^{r}+P(y)^{r}$ for all $x, y \in X$ and choosing $\alpha=2^{r-1}$ in Theorem 2.2, we get the desired result.

Theorem 2.4 Let $\varphi: X^{2} \rightarrow[0, \infty)$ be a function such that

$$
\Phi(x, y):=\sum_{j=0}^{\infty} \frac{1}{2^{j}} \varphi\left(2^{j} x, 2^{j} y\right)<\infty
$$

for all $x, y \in X$. Let $f: X \rightarrow Y$ be a mapping satisfying (2.2). Then there exists a unique Cauchy additive mapping $A: X \rightarrow Y$ such that

$$
\|f(x)-A(x)\| \leq \frac{1}{2} \Phi(x, x)
$$

for all $x \in X$.

Proof The proof is similar to the proof of [46, Theorem 2.2].

Remark 2.5 Let $r<1$. Letting $\varphi(x, y)=P(x)^{r}+P(y)^{r}$ for all $x, y \in X$ in Theorem 2.4, we obtain the inequality (2.6). The proof is given in [46, Theorem 2.2].

Theorem 2.6 Let $\varphi: Y^{2} \rightarrow[0, \infty)$ be a function such that there exists an $\alpha<1$ with

$$
\varphi(x, y) \leq \frac{\alpha}{2} \varphi(2 x, 2 y)
$$

for all $x, y \in Y$. Let $f: Y \rightarrow X$ be a mapping such that

$$
P(f(x+y)-f(x)-f(y)) \leq \varphi(x, y)
$$

for all $x, y \in Y$. Then there exists a unique additive mapping $A: Y \rightarrow X$ such that

$$
P(f(x)-A(x)) \leq \frac{\alpha}{2-2 \alpha} \varphi(x, x)
$$

for all $x \in Y$.

Proof Letting $y=x$ in (2.8), we get

$$
P(f(2 x)-2 f(x)) \leq \varphi(x, x),
$$

and so

$$
P\left(f(x)-2 f\left(\frac{x}{2}\right)\right) \leq \frac{\alpha}{2} \varphi(x, x)
$$

for all $x \in Y$.

Consider the set

$$
S:=\{h: Y \rightarrow X\}
$$


and introduce the generalized metric on $S$ :

$$
m(g, h)=\inf \left\{\mu \in \mathbb{R}_{+}: P(g(x)-h(x)) \leq \mu \varphi(x, x), \forall x \in Y\right\},
$$

where, as usual, $\inf \phi=+\infty$. It is easy to show that $(S, m)$ is complete (see [45, Lemma 2.1]).

Now we consider the linear mapping $J: S \rightarrow S$ such that

$$
J h(x):=2 h\left(\frac{x}{2}\right)
$$

for all $x \in Y$.

Let $g, h \in S$ be given such that $m(g, h)=\varepsilon$. Since

$$
P(J g(x)-J h(x))=P\left(2 g\left(\frac{x}{2}\right)-2 h\left(\frac{x}{2}\right)\right) \leq \alpha \varphi(x, x)
$$

for all $x \in Y, m(g, h)=\varepsilon$ implies that $m(J g, J h) \leq \alpha \varepsilon$. This means that

$$
m(J g, J h) \leq \alpha m(g, h)
$$

for all $g, h \in S$.

It follows from (2.10) that $m(f, J f) \leq \frac{\alpha}{2}$.

By Theorem 2.1, there exists a mapping $A: X \rightarrow Y$ satisfying the following:

(1) $A$ is a fixed point of $J$, i.e.,

$$
A\left(\frac{x}{2}\right)=\frac{1}{2} A(x)
$$

for all $x \in X$. The mapping $A$ is a unique fixed point of $J$ in the set

$$
M=\{g \in S: m(f, g)<\infty\} .
$$

This implies that $A$ is a unique mapping satisfying (2.11) such that there exists a $\mu \in(0, \infty)$ satisfying

$$
P(f(x)-A(x)) \leq \mu \varphi(x, x)
$$

for all $x \in Y$;

(2) $m\left(J^{n} f, A\right) \rightarrow 0$ as $n \rightarrow \infty$. This implies the equality

$$
\lim _{n \rightarrow \infty} 2^{n} f\left(\frac{x}{2^{n}}\right)=A(x)
$$

for all $x \in Y$;

(3) $m(f, A) \leq \frac{1}{1-\alpha} m(f, J f)$, which implies the inequality

$$
m(f, A) \leq \frac{\alpha}{2-2 \alpha} .
$$

This implies that the inequality (2.9) holds true. 
It follows from (2.7) and (2.8) that

$$
\begin{aligned}
P(A(x+y)-A(x)-A(y)) & =\lim _{n \rightarrow \infty} P\left(2^{n}\left(f\left(\frac{x+y}{2^{n}}\right)-f\left(\frac{x}{2^{n}}\right)-f\left(\frac{y}{2^{n}}\right)\right)\right) \\
& \leq \lim _{n \rightarrow \infty} 2^{n} P\left(\left(f\left(\frac{x+y}{2^{n}}\right)-f\left(\frac{x}{2^{n}}\right)-f\left(\frac{y}{2^{n}}\right)\right)\right) \\
& \leq \lim _{n \rightarrow \infty} \frac{2^{n} \alpha^{n}}{2^{n}} \varphi(x, y)=0
\end{aligned}
$$

for all $x, y \in Y$. So, $A(x+y)-A(x)-A(y)=0$ for all $x, y \in Y$. Thus $A: Y \rightarrow X$ is an additive mapping, as desired.

Corollary 2.7 Let $r, \theta$ be positive real numbers with $r>1$, and let $f: Y \rightarrow X$ be a mapping such that

$$
P(f(x+y)-f(x)-f(y)) \leq \theta\left(\|x\|^{r}+\|y\|^{r}\right)
$$

for all $x, y \in Y$. Then there exists a unique Cauchy additive mapping $A: Y \rightarrow X$ such that

$$
P(f(x)-A(x)) \leq \frac{2 \theta}{2^{r}-2}\|x\|^{r}
$$

for all $x \in Y$.

Proof Taking $\varphi(x, y)=\theta\left(\|x\|^{r}+\|y\|^{r}\right)$ for all $x, y \in X$ and choosing $\alpha=2^{1-r}$ in Theorem 2.6, we get the desired result.

Theorem 2.8 Let $\varphi: Y^{2} \rightarrow[0, \infty)$ be a function such that

$$
\Phi(x, y):=\sum_{j=1}^{\infty} 2^{j} \varphi\left(\frac{x}{2^{j}}, \frac{y}{2^{j}}\right)<\infty
$$

for all $x, y \in Y$. Let $f: Y \rightarrow X$ be a mapping satisfying (2.8). Then there exists a unique Cauchy additive mapping $A: Y \rightarrow X$ such that

$$
P(f(x)-A(x)) \leq \frac{1}{2} \Phi(x, x)
$$

for all $x \in Y$.

Proof The proof is similar to the proof of [46, Theorem 2.1].

Remark 2.9 Let $r>1$. Letting $\varphi(x, y)=\theta\left(\|x\|^{r}+\|y\|^{r}\right)$ for all $x, y \in X$ in Theorem 2.8, we obtain the inequality (2.12). The proof is given in [46, Theorem 2.1].

\section{Hyers-Ulam stability of the quadratic functional equation (1.1)}

Using the fixed point method and the direct method, we prove the Hyers-Ulam stability of the quadratic functional equation (1.1) in paranormed spaces. 
Theorem 3.1 Let $\varphi: X^{2} \rightarrow[0, \infty)$ be a function such that there exists an $\alpha<1$ with

$$
\varphi(x, y) \leq 4 \alpha \varphi\left(\frac{x}{2}, \frac{y}{2}\right)
$$

for all $x, y \in X$. Let $f: X \rightarrow Y$ be a mapping satisfying $f(0)=0$ and

$$
\|f(x+y)+f(x-y)-2 f(x)-2 f(y)\| \leq \varphi(x, y)
$$

for all $x, y \in X$. Then there exists a unique quadratic mapping $Q_{2}: X \rightarrow Y$ such that

$$
\left\|f(x)-Q_{2}(x)\right\| \leq \frac{1}{4-4 \alpha} \varphi(x, x)
$$

for all $x \in X$.

Proof Letting $y=x$ in (3.1), we get

$$
\|f(2 x)-4 f(x)\| \leq \varphi(x, x),
$$

and so

$$
\left\|f(x)-\frac{1}{4} f(2 x)\right\| \leq \frac{1}{4} \varphi(x, x)
$$

for all $x \in X$.

The rest of the proof is similar to the proof of Theorem 2.2.

Corollary 3.2 Let $r$ be a positive real number with $r<2$, and let $f: X \rightarrow Y$ be a mapping satisfying $f(0)=0$ and

$$
\|f(x+y)+f(x-y)-2 f(x)-2 f(y)\| \leq P(x)^{r}+P(y)^{r}
$$

for all $x, y \in X$. Then there exists a unique quadratic mapping $Q_{2}: X \rightarrow Y$ such that

$$
\left\|f(x)-Q_{2}(x)\right\| \leq \frac{2}{4-2^{r}} P(x)^{r}
$$

for all $x \in X$.

Proof Taking $\varphi(x, y)=P(x)^{r}+P(y)^{r}$ for all $x, y \in X$ and choosing $\alpha=2^{r-2}$ in Theorem 3.1, we get the desired result.

Theorem 3.3 Let $\varphi: X^{2} \rightarrow[0, \infty)$ be a function such that

$$
\Phi(x, y):=\sum_{j=0}^{\infty} \frac{1}{4^{j}} \varphi\left(2^{j} x, 2^{j} y\right)<\infty
$$

for all $x, y \in X$. Let $f: X \rightarrow Y$ be a mapping satisfying $f(0)=0$ and (3.1). Then there exists a unique quadratic mapping $Q_{2}: X \rightarrow Y$ such that

$$
\left\|f(x)-Q_{2}(x)\right\| \leq \frac{1}{4} \Phi(x, x)
$$

for all $x \in X$. 
Proof The proof is similar to the proof of [46, Theorem 3.2].

Remark 3.4 Let $r<2$. Letting $\varphi(x, y)=P(x)^{r}+P(y)^{r}$ for all $x, y \in X$ in Theorem 3.3, we obtain the inequality (3.2). The proof is given in [46, Theorem 3.2].

Theorem 3.5 Let $\varphi: Y^{2} \rightarrow[0, \infty)$ be a function such that there exists an $\alpha<1$ with

$$
\varphi(x, y) \leq \frac{\alpha}{4} \varphi(2 x, 2 y)
$$

for all $x, y \in Y$. Let $f: Y \rightarrow X$ be a mapping satisfying $f(0)=0$ and

$$
P(f(x+y)+f(x-y)-2 f(x)-2 f(y)) \leq \varphi(x, y)
$$

for all $x, y \in Y$. Then there exists a unique quadratic mapping $Q_{2}: Y \rightarrow X$ such that

$$
P\left(f(x)-Q_{2}(x)\right) \leq \frac{\alpha}{4-4 \alpha} \varphi(x, x)
$$

for all $x \in Y$.

Proof Letting $y=x$ in (3.3), we get

$$
P(f(2 x)-4 f(x)) \leq \varphi(x, x)
$$

and so

$$
P\left(f(x)-4 f\left(\frac{x}{2}\right)\right) \leq \varphi\left(\frac{x}{2}, \frac{x}{2}\right) \leq \frac{\alpha}{4} \varphi(x, x)
$$

for all $x \in Y$.

The rest of the proof is similar to the proof of Theorem 2.6.

Corollary 3.6 Let $r, \theta$ be positive real numbers with $r>2$, and let $f: Y \rightarrow X$ be a mapping satisfying $f(0)=0$ and

$$
P(f(x+y)+f(x-y)-2 f(x)-2 f(y)) \leq \theta\left(\|x\|^{r}+\|y\|^{r}\right)
$$

for all $x, y \in Y$. Then there exists a unique quadratic mapping $Q_{2}: Y \rightarrow X$ such that

$$
P\left(f(x)-Q_{2}(x)\right) \leq \frac{2 \theta}{2^{r}-4}\|x\|^{r}
$$

for all $x \in Y$.

Proof Taking $\varphi(x, y)=\theta\left(\|x\|^{r}+\|y\|^{r}\right)$ for all $x, y \in X$ and choosing $\alpha=2^{2-r}$ in Theorem 3.5, we get the desired result.

Theorem 3.7 Let $\varphi: Y^{2} \rightarrow[0, \infty)$ be a function such that

$$
\Phi(x, y):=\sum_{j=1}^{\infty} 4^{j} \varphi\left(\frac{x}{2^{j}}, \frac{y}{2^{j}}\right)<\infty
$$


for all $x, y \in Y$. Let $f: Y \rightarrow X$ be a mapping satisfying $f(0)=0$ and (3.3). Then there exists a unique quadratic mapping $Q_{2}: Y \rightarrow X$ such that

$$
P\left(f(x)-Q_{2}(x)\right) \leq \frac{1}{4} \Phi(x, x)
$$

for all $x \in Y$.

Proof The proof is similar to the proof of [46, Theorem 3.1].

Remark 3.8 Let $r>2$. Letting $\varphi(x, y)=\theta\left(\|x\|^{r}+\|y\|^{r}\right)$ for all $x, y \in X$ in Theorem 3.7, we obtain the inequality (3.4). The proof is given in [46, Theorem 3.1].

\section{Hyers-Ulam stability of the cubic functional equation (1.2)}

Using the fixed point method and the direct method, we prove the Hyers-Ulam stability of the cubic functional equation (1.2) in paranormed spaces.

Theorem 4.1 Let $\varphi: X^{2} \rightarrow[0, \infty)$ be a function such that there exists an $\alpha<1$ with

$$
\varphi(x, y) \leq 8 \alpha \varphi\left(\frac{x}{2}, \frac{y}{2}\right)
$$

for all $x, y \in X$. Let $f: X \rightarrow Y$ be a mapping such that

$$
\left\|\frac{1}{2} f(2 x+y)+\frac{1}{2} f(2 x-y)-f(x+y)-f(x-y)-6 f(x)\right\| \leq \varphi(x, y)
$$

for all $x, y \in X$. Then there exists a unique cubic mapping $C: X \rightarrow Y$ such that

$$
\|f(x)-C(x)\| \leq \frac{1}{8-8 \alpha} \varphi(x, 0)
$$

for all $x \in X$.

Proof Letting $y=0$ in (4.1), we get

$$
\|f(2 x)-8 f(x)\| \leq \varphi(x, 0)
$$

and so

$$
\left\|f(x)-\frac{1}{8} f(2 x)\right\| \leq \frac{1}{8} \varphi(x, 0)
$$

for all $x \in X$.

The rest of the proof is similar to the proof of Theorem 2.2.

Corollary 4.2 Let $r$ be a positive real number with $r<3$, and let $f: Y \rightarrow X$ be a mapping such that

$$
\left\|\frac{1}{2} f(2 x+y)+\frac{1}{2} f(2 x-y)-f(x+y)-f(x-y)-6 f(x)\right\| \leq P(x)^{r}+P(y)^{r}
$$


for all $x, y \in X$. Then there exists a unique cubic mapping $C: Y \rightarrow X$ such that

$$
P(f(x)-C(x)) \leq \frac{1}{8-2^{r}} P(x)^{r}
$$

for all $x \in Y$.

Proof Taking $\varphi(x, y)=P(x)^{r}+P(y)^{r}$ for all $x, y \in X$ and choosing $\alpha=2^{r-3}$ in Theorem 4.1, we get the desired result.

Theorem 4.3 Let $\varphi: X^{2} \rightarrow[0, \infty)$ be a function such that

$$
\Phi(x, y):=\sum_{j=0}^{\infty} \frac{1}{8^{j}} \varphi\left(2^{j} x, 2^{j} y\right)<\infty
$$

for all $x, y \in X$. Letf $: X \rightarrow Y$ be a mapping satisfying (4.1). Then there exists a unique cubic mapping $C: X \rightarrow Y$ such that

$$
\|f(x)-C(x)\| \leq \frac{1}{8} \Phi(x, 0)
$$

for all $x \in X$.

Proof The proof is similar to the proof of [46, Theorem 4.2].

Remark 4.4 Let $r<3$. Letting $\varphi(x, y)=P(x)^{r}+P(y)^{r}$ for all $x, y \in X$ in Theorem 4.3, we obtain the inequality (4.2). The proof is given in [46, Theorem 4.2].

Theorem 4.5 Let $\varphi: Y^{2} \rightarrow[0, \infty)$ be a function such that there exists an $\alpha<1$ with

$$
\varphi(x, y) \leq \frac{\alpha}{8} \varphi(2 x, 2 y)
$$

for all $x, y \in Y$. Let $f: Y \rightarrow X$ be a mapping such that

$$
P\left(\frac{1}{2} f(2 x+y)+\frac{1}{2} f(2 x-y)-f(x+y)-f(x-y)-6 f(x)\right) \leq \varphi(x, y)
$$

for all $x, y \in Y$. Then there exists a unique cubic mapping $C: Y \rightarrow X$ such that

$$
P(f(x)-C(x)) \leq \frac{\alpha}{8-8 \alpha} \varphi(x, 0)
$$

for all $x \in Y$.

Proof Letting $y=0$ in (4.3), we get

$$
P(f(2 x)-8 f(x)) \leq \varphi(x, 0),
$$

and so

$$
P\left(f(x)-8 f\left(\frac{x}{2}\right)\right) \leq \varphi\left(\frac{x}{2}, \frac{x}{2}\right) \leq \frac{\alpha}{8} \varphi(x, 0)
$$

for all $x \in Y$. 
The rest of the proof is similar to the proof of Theorem 2.6.

Corollary 4.6 Let $r, \theta$ be positive real numbers with $r>3$, and let $f: Y \rightarrow X$ be a mapping such that

$$
P\left(\frac{1}{2} f(2 x+y)+\frac{1}{2} f(2 x-y)-f(x+y)-f(x-y)-6 f(x)\right) \leq \theta\left(\|x\|^{r}+\|y\|^{r}\right)
$$

for all $x, y \in Y$. Then there exists a unique cubic mapping $C: Y \rightarrow X$ such that

$$
P(f(x)-C(x)) \leq \frac{\theta}{2^{r}-8}\|x\|^{r}
$$

for all $x \in Y$.

Proof Taking $\varphi(x, y)=\theta\left(\|x\|^{r}+\|y\|^{r}\right)$ for all $x, y \in X$ and choosing $\alpha=2^{3-r}$ in Theorem 4.5, we get the desired result.

Theorem 4.7 Let $\varphi: Y^{2} \rightarrow[0, \infty)$ be a function such that

$$
\Phi(x, y):=\sum_{j=1}^{\infty} 8^{j} \varphi\left(\frac{x}{2^{j}}, \frac{y}{2^{j}}\right)<\infty
$$

for all $x, y \in Y$. Let $f: Y \rightarrow X$ be a mapping satisfying (4.3). Then there exists a unique cubic mapping $C: Y \rightarrow X$ such that

$$
P(f(x)-C(x)) \leq \frac{1}{8} \Phi(x, 0)
$$

for all $x \in Y$.

Proof The proof is similar to the proof of [46, Theorem 4.1].

Remark 4.8 Let $r>3$. Letting $\varphi(x, y)=\theta\left(\|x\|^{r}+\|y\|^{r}\right)$ for all $x, y \in X$ in Theorem 4.7, we obtain the inequality (4.4). The proof is given in [46, Theorem 4.1].

\section{Hyers-Ulam stability of the quartic functional equation (1.3)}

Using the fixed point method and the direct method, we prove the Hyers-Ulam stability of the quartic functional equation (1.3) in paranormed spaces.

Theorem 5.1 Let $\varphi: X^{2} \rightarrow[0, \infty)$ be a function such that there exists an $\alpha<1$ with

$$
\varphi(x, y) \leq 16 \alpha \varphi\left(\frac{x}{2}, \frac{y}{2}\right)
$$

for all $x, y \in X$. Let $f: X \rightarrow Y$ be a mapping satisfying $f(0)=0$ and

$$
\left\|\frac{1}{2} f(2 x+y)+\frac{1}{2} f(2 x-y)-2 f(x+y)-2 f(x-y)-12 f(x)+3 f(y)\right\| \leq \varphi(x, y)
$$


for all $x, y \in X$. Then there exists a unique quartic mapping $Q_{4}: X \rightarrow Y$ such that

$$
\left\|f(x)-Q_{4}(x)\right\| \leq \frac{1}{16-16 \alpha} \varphi(x, 0)
$$

for all $x \in X$.

Proof Letting $y=0$ in (5.1), we get

$$
\|f(2 x)-16 f(x)\| \leq \varphi(x, 0),
$$

and so

$$
\left\|f(x)-\frac{1}{16} f(2 x)\right\| \leq \frac{1}{16} \varphi(x, 0)
$$

for all $x \in X$.

The rest of the proof is similar to the proof of Theorem 2.2.

Corollary 5.2 Let $r$ be a positive real number with $r<4$, and let $f: Y \rightarrow X$ be a mapping satisfying $f(0)=0$ and

$$
\left\|\frac{1}{2} f(2 x+y)+\frac{1}{2} f(2 x-y)-2 f(x+y)-2 f(x-y)-12 f(x)+3 f(y)\right\| \leq P(x)^{r}+P(y)^{r}
$$

for all $x, y \in X$. Then there exists a unique quartic mapping $Q_{4}: Y \rightarrow X$ such that

$$
P\left(f(x)-Q_{4}(x)\right) \leq \frac{1}{16-2^{r}} P(x)^{r}
$$

for all $x \in Y$.

Proof Taking $\varphi(x, y)=P(x)^{r}+P(y)^{r}$ for all $x, y \in X$ and choosing $\alpha=2^{r-4}$ in Theorem 5.1, we get the desired result.

Theorem 5.3 Let $\varphi: X^{2} \rightarrow[0, \infty)$ be a function such that

$$
\Phi(x, y):=\sum_{j=0}^{\infty} \frac{1}{16^{j}} \varphi\left(2^{j} x, 2^{j} y\right)<\infty
$$

for all $x, y \in X$. Let $f: X \rightarrow Y$ be a mapping satisfying $f(0)=0$ and (5.1). Then there exists a unique quartic mapping $Q_{4}: X \rightarrow Y$ such that

$$
\left\|f(x)-Q_{4}(x)\right\| \leq \frac{1}{16} \Phi(x, 0)
$$

for all $x \in X$.

Proof The proof is similar to the proof of [46, Theorem 5.2].

Remark 5.4 Let $r<4$. Letting $\varphi(x, y)=P(x)^{r}+P(y)^{r}$ for all $x, y \in X$ in Theorem 5.3, we obtain the inequality (5.2). The proof is given in [46, Theorem 5.2]. 
Theorem 5.5 Let $\varphi: Y^{2} \rightarrow[0, \infty)$ be a function such that there exists an $\alpha<1$ with

$$
\varphi(x, y) \leq \frac{\alpha}{16} \varphi(2 x, 2 y)
$$

for all $x, y \in Y$. Let $f: Y \rightarrow X$ be a mapping satisfying $f(0)=0$ and

$$
P\left(\frac{1}{2} f(2 x+y)+\frac{1}{2} f(2 x-y)-2 f(x+y)-2 f(x-y)-12 f(x)+3 f(y)\right) \leq \varphi(x, y)
$$

for all $x, y \in Y$. Then there exists a unique quartic mapping $Q_{4}: Y \rightarrow X$ such that

$$
P\left(f(x)-Q_{4}(x)\right) \leq \frac{\alpha}{16-16 \alpha} \varphi(x, 0)
$$

for all $x \in Y$.

Proof Letting $y=0$ in (5.3), we get

$$
P(f(2 x)-16 f(x)) \leq \varphi(x, 0)
$$

and so

$$
P\left(f(x)-16 f\left(\frac{x}{2}\right)\right) \leq \varphi\left(\frac{x}{2}, \frac{x}{2}\right) \leq \frac{\alpha}{16} \varphi(x, 0)
$$

for all $x \in Y$.

The rest of the proof is similar to the proof of Theorem 2.6.

Corollary 5.6 Let $r, \theta$ be positive real numbers with $r>4$, and let $f: Y \rightarrow X$ be a mapping satisfying $f(0)=0$ and

$$
P\left(\frac{1}{2} f(2 x+y)+\frac{1}{2} f(2 x-y)-2 f(x+y)-2 f(x-y)-12 f(x)+3 f(y)\right) \leq \theta\left(\|x\|^{r}+\|y\|^{r}\right)
$$

for all $x, y \in Y$. Then there exists a unique quartic mapping $Q_{4}: Y \rightarrow X$ such that

$$
P\left(f(x)-Q_{4}(x)\right) \leq \frac{\theta}{2^{r}-16}\|x\|^{r}
$$

for all $x \in Y$.

Proof Taking $\varphi(x, y)=\theta\left(\|x\|^{r}+\|y\|^{r}\right)$ for all $x, y \in X$ and choosing $\alpha=2^{4-r}$ in Theorem 5.5, we get the desired result.

Theorem 5.7 Let $\varphi: Y^{2} \rightarrow[0, \infty)$ be a function such that

$$
\Phi(x, y):=\sum_{j=1}^{\infty} 16^{j} \varphi\left(\frac{x}{2^{j}}, \frac{y}{2^{j}}\right)<\infty
$$


for all $x, y \in Y$. Let $f: Y \rightarrow X$ be a mapping satisfying $f(0)=0$ and (5.3). Then there exists a unique quartic mapping $Q_{4}: Y \rightarrow X$ such that

$$
P\left(f(x)-Q_{4}(x)\right) \leq \frac{1}{16} \Phi(x, 0)
$$

for all $x \in Y$.

Proof The proof is similar to the proof of [46, Theorem 5.1].

Remark 5.8 Let $r>4$. Letting $\varphi(x, y)=\theta\left(\|x\|^{r}+\|y\|^{r}\right)$ for all $x, y \in X$ in Theorem 5.7, we obtain the inequality (5.4). The proof is given in [46, Theorem 5.1].

\section{Stability of a functional inequality associated with a three-variable Jensen additive functional equation}

Using the fixed point method and the direct method, we prove the Hyers-Ulam stability of a functional inequality associated with a Jordan-von Neumann type three-variable Jensen additive functional equation in paranormed spaces.

Proposition 6.1 [36, Proposition 2.1] Let $f: X \rightarrow Y$ be a mapping such that

$$
\|f(x)+f(y)+f(z)\| \leq\left\|2 f\left(\frac{x+y+z}{2}\right)\right\|
$$

for all $x, y, z \in X$. Then $f$ is Cauchy additive.

Theorem 6.2 Let $\varphi: X^{3} \rightarrow[0, \infty)$ be a function such that there exists an $\alpha<1$ with

$$
\varphi(x, y, z) \leq 2 \alpha \varphi\left(\frac{x}{2}, \frac{y}{2}, \frac{z}{2}\right)
$$

for all $x, y, z \in X$. Let $f: X \rightarrow Y$ be an odd mapping such that

$$
\|f(x)+f(y)+f(z)\| \leq\left\|2 f\left(\frac{x+y+z}{2}\right)\right\|+\varphi(x, y, z)
$$

for all $x, y, z \in X$. Then there exists a unique Cauchy additive mapping $A: X \rightarrow Y$ such that

$$
\|f(x)-A(x)\| \leq \frac{1}{2-2 \alpha} \varphi(x, x,-2 x)
$$

for all $x \in X$.

Proof Letting $y=x$ and $z=-2 x$ in (6.2), we get

$$
\|2 f(x)-f(2 x)\|=\|2 f(x)+f(-2 x)\| \leq \varphi(x, x,-2 x),
$$

and so

$$
\left\|f(x)-\frac{1}{2} f(2 x)\right\| \leq \frac{1}{2} \varphi(x, x,-2 x)
$$

for all $x \in X$. 
Consider the set

$$
S:=\{h: X \rightarrow Y\}
$$

and introduce the generalized metric on $S$

$$
m(g, h)=\inf \left\{\mu \in \mathbb{R}_{+}:\|g(x)-h(x)\| \leq \mu \varphi(x, x,-2 x), \forall x \in X\right\},
$$

where, as usual, inf $\phi=+\infty$. It is easy to show that $(S, m)$ is complete (see [45, Lemma 2.1]).

Now we consider the linear mapping $J: S \rightarrow S$ such that

$$
J h(x):=\frac{1}{2} h(2 x)
$$

for all $x \in X$.

Let $g, h \in S$ be given such that $m(g, h)=\varepsilon$. Since

$$
\|J g(x)-J h(x)\|=\left\|\frac{1}{2} g(2 x)-\frac{1}{2} h(2 x)\right\| \leq \alpha \varphi(x, x,-2 x)
$$

for all $x \in X, m(g, h)=\varepsilon$ implies that $m(J g, J h) \leq \alpha \varepsilon$. This means that

$$
m(J g, J h) \leq \alpha m(g, h)
$$

for all $g, h \in S$.

It follows from (6.4) that $m(f, J f) \leq \frac{1}{2}$.

By Theorem 2.1, there exists a mapping $A: X \rightarrow Y$ satisfying the following:

(1) $A$ is a fixed point of $J$, i.e.,

$$
A(2 x)=2 A(x)
$$

for all $x \in X$. The mapping $A$ is a unique fixed point of $J$ in the set

$$
M=\{g \in S: m(f, g)<\infty\} .
$$

This implies that $A$ is a unique mapping satisfying (6.5) such that there exists a $\mu \in(0, \infty)$ satisfying

$$
\|f(x)-A(x)\| \leq \mu \varphi(x, x,-2 x)
$$

for all $x \in X$;

(2) $m\left(J^{n} f, A\right) \rightarrow 0$ as $n \rightarrow \infty$. This implies the equality

$$
\lim _{n \rightarrow \infty} \frac{1}{2^{n}} f\left(2^{n} x\right)=A(x)
$$

for all $x \in X$; 
(3) $m(f, A) \leq \frac{1}{1-\alpha} m(f, J f)$, which implies the inequality

$$
m(f, A) \leq \frac{1}{2-2 \alpha}
$$

This implies that the inequality (6.3) holds true.

It follows from (6.1) and (6.2) that

$$
\begin{aligned}
\frac{1}{2^{n}}\left\|f\left(2^{n} x\right)+f\left(2^{n} y\right)+f\left(2^{n} z\right)\right\| & \leq \frac{1}{2^{n}}\left\|2 f\left(\frac{2^{n}(x+y+z)}{2}\right)\right\|+\frac{1}{2^{n}} \varphi\left(2^{n} x, 2^{n} y, 2^{n} z\right) \\
& \leq \frac{1}{2^{n}}\left\|2 f\left(\frac{2^{n}(x+y+z)}{2}\right)\right\|+\frac{2^{n} \alpha^{n}}{2^{n}} \varphi(x, y, z)
\end{aligned}
$$

for all $x, y, z \in X$. Letting $n \rightarrow \infty$ in (6.6), we get

$$
\|A(x)+A(y)+A(z)\| \leq\left\|2 A\left(\frac{x+y+z}{2}\right)\right\|
$$

for all $x, y, z \in X$. By Proposition 6.1, $A: X \rightarrow Y$ is Cauchy additive, as desired.

Corollary 6.3 [47, Theorem 2.2] Let $r$ be a positive real number with $r<1$, and let $f: X \rightarrow$ $Y$ be an odd mapping such that

$$
\|f(x)+f(y)+f(z)\| \leq\left\|2 f\left(\frac{x+y+z}{2}\right)\right\|+P(x)^{r}+P(y)^{r}+P(z)^{r}
$$

for all $x, y, z \in X$. Then there exists a unique Cauchy additive mapping $A: X \rightarrow Y$ such that

$$
\|f(x)-A(x)\| \leq \frac{2+2^{r}}{2-2^{r}} P(x)^{r}
$$

for all $x \in X$.

Proof Taking $\varphi(x, y, z)=P(x)^{r}+P(y)^{r}+P(z)^{r}$ for all $x, y, z \in X$ and choosing $\alpha=2^{r-1}$ in Theorem 6.2, we get the desired result.

Theorem 6.4 Let $\varphi: X^{3} \rightarrow[0, \infty)$ be a function such that

$$
\Phi(x, y, z):=\sum_{j=0}^{\infty} \frac{1}{2^{j}} \varphi\left(2^{j} x, 2^{j} y, 2^{j} z\right)<\infty
$$

for all $x, y, z \in X$. Let $f: X \rightarrow Y$ be an odd mapping satisfying (6.2). Then there exists a unique Cauchy additive mapping $A: X \rightarrow Y$ such that

$$
\|f(x)-A(x)\| \leq \frac{1}{2} \Phi(x, x,-2 x)
$$

for all $x \in X$.

Proof The proof is similar to the proof of [47, Theorem 2.2].

Remark 6.5 Let $r<1$. Letting $\varphi(x, y, z)=P(x)^{r}+P(y)^{r}+P(z)^{r}$ for all $x, y \in X$ in Theorem 6.4, we obtain the inequality (6.7). The proof is given in [47, Theorem 2.2]. 


\section{Stability of a functional inequality associated with a three-variable Cauchy additive functional equation}

Using the fixed point method and the direct method, we prove the Hyers-Ulam stability of a functional inequality associated with a Jordan-von Neumann type three-variable Cauchy additive functional equation in paranormed spaces.

Proposition 7.1 [36, Proposition 2.2] Let $f: X \rightarrow Y$ be a mapping such that

$$
\|f(x)+f(y)+f(z)\| \leq\|f(x+y+z)\|
$$

for all $x, y, z \in X$. Then $f$ is Cauchy additive.

Theorem 7.2 Let $\varphi: X^{3} \rightarrow[0, \infty)$ be a function such that there exists an $\alpha<1$ with

$$
\varphi(x, y, z) \leq 2 \alpha \varphi\left(\frac{x}{2}, \frac{y}{2}, \frac{z}{2}\right)
$$

for all $x, y, z \in X$. Let $f: X \rightarrow Y$ be an odd mapping such that

$$
\|f(x)+f(y)+f(z)\| \leq\|f(x+y+z)\|+\varphi(x, y, z)
$$

for all $x, y, z \in X$. Then there exists a unique Cauchy additive mapping $A: X \rightarrow Y$ such that

$$
\|f(x)-A(x)\| \leq \frac{1}{2-2 \alpha} \varphi(x, x,-2 x)
$$

for all $x \in X$.

Proof Letting $y=x$ and $z=-2 x$ in (7.1), we get

$$
\|2 f(x)-f(2 x)\|=\|2 f(x)+f(-2 x)\| \leq \varphi(x, x,-2 x),
$$

and so

$$
\left\|f(x)-\frac{1}{2} f(2 x)\right\| \leq \frac{1}{2} \varphi(x, x,-2 x)
$$

for all $x \in X$.

The rest of the proof is similar to the proof of Theorem 6.2.

Corollary 7.3 [47, Theorem 3.2] Let $r$ be a positive real number with $r<1$, and let $f: X \rightarrow$ $Y$ be an odd mapping such that

$$
\|f(x)+f(y)+f(z)\| \leq\|f(x+y+z)\|+P(x)^{r}+P(y)^{r}+P(z)^{r}
$$

for all $x, y, z \in X$. Then there exists a unique Cauchy additive mapping $A: X \rightarrow Y$ such that

$$
\|f(x)-A(x)\| \leq \frac{2+2^{r}}{2-2^{r}} P(x)^{r}
$$

for all $x \in X$. 
Proof Taking $\varphi(x, y, z)=P(x)^{r}+P(y)^{r}+P(z)^{r}$ for all $x, y, z \in X$ and choosing $\alpha=2^{r-1}$ in Theorem 7.2, we get the desired result.

Theorem 7.4 Let $\varphi: X^{3} \rightarrow[0, \infty)$ be a function such that

$$
\Phi(x, y, z):=\sum_{j=0}^{\infty} \frac{1}{2^{j}} \varphi\left(2^{j} x, 2^{j} y, 2^{j} z\right)<\infty
$$

for all $x, y, z \in X$. Let $f: X \rightarrow Y$ be an odd mapping satisfying (7.1). Then there exists a unique Cauchy additive mapping $A: X \rightarrow Y$ such that

$$
\|f(x)-A(x)\| \leq \frac{1}{2} \Phi(x, x,-2 x)
$$

for all $x \in X$.

Proof The proof is similar to the proof of [47, Theorem 3.2].

Remark7.5 Let $r<1$. Letting $\varphi(x, y, z)=P(x)^{r}+P(y)^{r}+P(z)^{r}$ for all $x, y \in X$ in Theorem 7.4, we obtain the inequality (7.3). The proof is given in [47, Theorem 3.2].

\section{Stability of a functional inequality associated with the Cauchy-Jensen functional equation}

Using the fixed point method and the direct method, we prove the Hyers-Ulam stability of a functional inequality associated with a Jordan-von Neumann type Cauchy-Jensen additive functional equation in paranormed spaces.

Proposition 8.1 [36, Proposition 2.3] Let $f: X \rightarrow Y$ be a mapping such that

$$
\|f(x)+f(y)+2 f(z)\| \leq\left\|2 f\left(\frac{x+y}{2}+z\right)\right\|
$$

for all $x, y, z \in X$. Then $f$ is Cauchy additive.

Theorem 8.2 Let $\varphi: X^{3} \rightarrow[0, \infty)$ be a function such that there exists an $\alpha<1$ with

$$
\varphi(x, y, z) \leq 2 \alpha \varphi\left(\frac{x}{2}, \frac{y}{2}, \frac{z}{2}\right)
$$

for all $x, y, z \in X$. Let $f: X \rightarrow Y$ be an odd mapping such that

$$
\|f(x)+f(y)+2 f(z)\| \leq\left\|2 f\left(\frac{x+y}{2}+z\right)\right\|+\varphi(x, y, z)
$$

for all $x, y, z \in X$. Then there exists a unique Cauchy additive mapping $A: X \rightarrow Y$ such that

$$
\|f(x)-A(x)\| \leq \frac{1}{2-2 \alpha} \varphi(2 x, 0,-x)
$$

for all $x \in X$. 
Proof Replacing $x$ by $2 x$ and letting $y=0$ and $z=-x$ in (8.1), we get

$$
\|2 f(x)-f(2 x)\|=\|2 f(x)+f(-2 x)\| \leq \varphi(2 x, 0,-x)
$$

for all $x \in X$.

The rest of the proof is the same as in the proof of Theorem 6.2.

Corollary 8.3 [47, Theorem 4.2] Let $r$ be a positive real number with $r<1$, and let $f: X \rightarrow$ $Y$ be an odd mapping such that

$$
\|f(x)+f(y)+2 f(z)\| \leq\left\|2 f\left(\frac{x+y}{2}+z\right)\right\|+P(x)^{r}+P(y)^{r}+P(z)^{r}
$$

for all $x, y, z \in X$. Then there exists a unique Cauchy additive mapping $A: X \rightarrow Y$ such that

$$
\|f(x)-A(x)\| \leq \frac{1+2^{r}}{2-2^{r}} P(x)^{r}
$$

for all $x \in X$.

Proof Taking $\varphi(x, y, z)=P(x)^{r}+P(y)^{r}+P(z)^{r}$ for all $x, y, z \in X$ and choosing $\alpha=2^{r-1}$ in Theorem 8.2, we get the desired result.

Theorem 8.4 Let $\varphi: X^{3} \rightarrow[0, \infty)$ be a function such that

$$
\Phi(x, y, z):=\sum_{j=0}^{\infty} \frac{1}{2^{j}} \varphi\left(2^{j} x, 2^{j} y, 2^{j} z\right)<\infty
$$

for all $x, y, z \in X$. Let $f: X \rightarrow Y$ be an odd mapping satisfying (8.1). Then there exists a unique Cauchy additive mapping $A: X \rightarrow Y$ such that

$$
\|f(x)-A(x)\| \leq \frac{1}{2} \Phi(2 x, 0,-x)
$$

for all $x \in X$.

Proof The proof is similar to the proof of [47, Theorem 4.2].

Remark 8.5 Let $r<1$. Letting $\varphi(x, y, z)=P(x)^{r}+P(y)^{r}+P(z)^{r}$ for all $x, y \in X$ in Theorem 8.4, we obtain the inequality (8.3). The proof is given in [47, Theorem 4.2]. 


\section{Acknowledgements}

This work was supported by the Daejin University Research Grant in 2013.

Received: 3 September 2012 Accepted: 8 March 2013 Published: 22 April 2013

\section{References}

1. Fast, H: Sur la convergence statistique. Colloq. Math. 2, 241-244 (1951)

2. Steinhaus, H: Sur la convergence ordinaire et la convergence asymptotique. Colloq. Math. 2, $73-74$ (1951)

3. Fridy, JA: On statistical convergence. Analysis 5, 301-313 (1985)

4. Karakus, S: Statistical convergence on probabilistic normed spaces. Math. Commun. 12, 11-23 (2007)

5. Mursaleen, M: $\boldsymbol{\lambda}$-statistical convergence. Math. Slovaca 50,111-115 (2000)

6. Mursaleen, M, Mohiuddine, SA: On lacunary statistical convergence with respect to the intuitionistic fuzzy normed space. J. Comput. Appl. Math. 233, 142-149 (2009)

7. Šalát, T: On the statistically convergent sequences of real numbers. Math. Slovaca 30, 139-150 (1980)

8. Kolk, E: The statistical convergence in Banach spaces. Tartu ülik. Toim. 928, 41-52 (1991)

9. Wilansky, A: Modern Methods in Topological Vector Space. McGraw-Hill, New York (1978)

10. Ulam, SM: A Collection of the Mathematical Problems. Interscience, New York (1960)

11. Hyers, DH: On the stability of the linear functional equation. Proc. Natl. Acad. Sci. USA 27, $222-224$ (1941)

12. Aoki, T: On the stability of the linear transformation in Banach spaces. J. Math. Soc. Jpn. 2, 64-66 (1950)

13. Rassias, TM: On the stability of the linear mapping in Banach spaces. Proc. Am. Math. Soc. 72, $297-300$ (1978)

14. Găvruta, P: A generalization of the Hyers-Ulam-Rassias stability of approximately additive mappings. J. Math. Anal. Appl. 184, 431-436 (1994)

15. Rassias, TM: Problem 16; 2. Report of the 27th International Symp. on Functional Equations, Aequationes Math. 39, 292-293; 309 (1990)

16. Gajda, Z: On stability of additive mappings. Int. J. Math. Math. Sci. 14, 431-434 (1991)

17. Rassias, TM, Šemrl, P: On the behaviour of mappings which do not satisfy Hyers-Ulam stability. Proc. Am. Math. Soc. 114, 989-993 (1992)

18. Czerwik, P: Functional Equations and Inequalities in Several Variables. World Scientific, Singapore (2002)

19. Hyers, DH, Isac, G, Rassias, TM: Stability of Functional Equations in Several Variables. Birkhäuser, Basel (1998)

20. Skof, F: Proprietà locali e approssimazione di operatori. Rend. Semin. Mat. Fis. Milano 53, 113-129 (1983)

21. Cholewa, PW: Remarks on the stability of functional equations. Aequ. Math. 27, 76-86 (1984)

22. Czerwik, S: On the stability of the quadratic mapping in normed spaces. Abh. Math. Semin. Univ. Hamb. 62, 59-64 (1992)

23. Aczel, J, Dhombres, J: Functional Equations in Several Variables. Cambridge University Press, Cambridge (1989)

24. Czerwik, S: Stability of Functional Equations of Ulam-Hyers-Rassias Type. Hadronic Press, Palm Harbor (2003)

25. Eshaghi Gordji, M, Savadkouhi, MB: Stability of a mixed type cubic-quartic functional equation in non-Archimedean spaces. Appl. Math. Lett. 23, 1198-1202 (2010)

26. Isac, G, Rassias, TM: On the Hyers-Ulam stability of $\psi$-additive mappings. J. Approx. Theory 72, 131-137 (1993)

27. Jun, K, Lee, Y: A generalization of the Hyers-Ulam-Rassias stability of the Pexiderized quadratic equations. J. Math. Anal. Appl. 297, 70-86 (2004)

28. Jung, S: Hyers-Ulam-Rassias Stability of Functional Equations in Mathematical Analysis. Hadronic Press, Palm Harbor (2001)

29. Park, C: Homomorphisms between Poisson JC*-algebras. Bull. Braz. Math. Soc. 36, 79-97 (2005)

30. Jun, K, Kim, H: The generalized Hyers-Ulam-Rassias stability of a cubic functional equation. J. Math. Anal. Appl. 274, 867-878 (2002)

31. Lee, S, Im, S, Hwang, I: Quartic functional equations. J. Math. Anal. Appl. 307, 387-394 (2005)

32. Gilányi, A: Eine zur Parallelogrammgleichung äquivalente Ungleichung. Aequ. Math. 62, 303-309 (2001)

33. Rätz, J: On inequalities associated with the Jordan-von Neumann functional equation. Aequ. Math. 66, 191-200 (2003)

34. Fechner, W: Stability of a functional inequalities associated with the Jordan-von Neumann functional equation. Aequ. Math. 71, 149-161 (2006)

35. Gilányi, A: On a problem by K. Nikodem. Math. Inequal. Appl. 5, 707-710 (2002)

36. Park, C, Cho, Y, Han, H: Functional inequalities associated with Jordan-von Neumann-type additive functional equations. J. Inequal. Appl. 2007, Article ID 41820 (2007)

37. Cădariu, L, Radu, V: Fixed points and the stability of Jensen's functional equation. J. Inequal. Pure Appl. Math. 4(1), Article ID 4 (2003)

38. Diaz, J, Margolis, B: A fixed point theorem of the alternative for contractions on a generalized complete metric space. Bull. Am. Math. Soc. 74, 305-309 (1968)

39. Isac, G, Rassias, TM: Stability of $\psi$-additive mappings: applications to nonlinear analysis. Int. J. Math. Math. Sci. 19 219-228 (1996)

40. Cădariu, L, Radu, V: On the stability of the Cauchy functional equation: a fixed point approach. Grazer Math. Ber. 346 , 43-52 (2004)

41. Cădariu, L, Radu, V: Fixed point methods for the generalized stability of functional equations in a single variable. Fixed Point Theory Appl. 2008, Article ID 749392 (2008)

42. Park, C: Fixed points and Hyers-Ulam-Rassias stability of Cauchy-Jensen functional equations in Banach algebras. Fixed Point Theory Appl. 2007, Article ID 50175 (2007)

43. Park, C: Generalized Hyers-Ulam-Rassias stability of quadratic functional equations: a fixed point approach. Fixed Point Theory Appl. 2008, Article ID 493751 (2008)

44. Radu, V: The fixed point alternative and the stability of functional equations. Fixed Point Theory 4, 91-96 (2003)

45. Miheț, D, Radu, V: On the stability of the additive Cauchy functional equation in random normed spaces. J. Math. Anal. Appl. 343, 567-572 (2008)

46. Park, C, Shin, D: Functional equations in paranormed spaces. Adv. Differ. Equ. 2012, Article ID 123 (2012)

47. Lee, S, Park, C, Lee, J: Functional inequalities in paranormed spaces. J. Chungcheong Math. Soc. 26, 287-296 (2013) 
doi:10.1186/1029-242X-2013-198

Cite this article as: Park and Lee: Functional equations and inequalities in paranormed spaces. Journal of Inequalities and Applications 2013 2013:198.

Submit your manuscript to a SpringerOpen ${ }^{\circ}$ journal and benefit from:

- Convenient online submission

- Rigorous peer review

- Immediate publication on acceptance

Open access: articles freely available online

- High visibility within the field

- Retaining the copyright to your article

Submit your next manuscript at $\gg$ springeropen.com 